\title{
PROCEDIMENTO NUMÉRICO DE PAINÉIS DE ALVENARIA
}

\author{
Lucas Henrique Pereira Silva; Flávia Aparecida Catuchi; Daniele Araujo Altran
}

Faculdade de Engenharia da Universidade do Oeste Paulista - UNOESTE, Presidente Prudente, São Paulo.E-mail: lucahpsilva@outlook.com

\section{RESUMO}

A Engenharia de Estruturas é uma área de grande importância na construção civil, uma vez que busca otimização de projetos. Diante desse fato, a alvenaria estrutural é um sistema que satisfaz essa procura, devido à agilidade na construção, com projetos modulados, equipe e equipamentos especializados, que garantem rapidez e diminuição de custos. Neste cenário, a modelagem numérica e a análise de tensões e deformações na alvenaria estrutural é muito importante, pois as características da alvenaria pode ser fator determinante na resistência do sistema. Desse modo, pretende-se apresentar com este trabalho um estudo de comparações de tensões e deformações em painéis de alvenaria estrutural, submetidos a esforços externos. Este estudo será viabilizado através de uma modelagem numérica desenvolvida em um programa estrutural existente no mercado e comparado com outro estudo já realizado previamente por outro autor o que permitirá comparações. Mesmo com as diferenças nas discretizações dos elementos e entre os programas utilizados os resultados encontrados foram satisfatórios, de modo que as tensões e deformações apresentaram comportamentos próximos ao já conseguido pela bibliografia de referência.

Palavras-chave: Alvenaria Estrutural; Tensões; Deformações; Painéis.

\section{INTRODUÇÃO}

A alvenaria estrutural é um dos sistemas construtivos existentes, mais antigos, empregado em construções de edificações e monumentos, desde a antiguidade.

A definição de alvenaria armada de blocos de concreto, segundo a Associação Brasileira de Normas Técnicas - ABNT (1989, p.2), é “aquela constituída com blocos vazados de concreto, assentados com argamassa, na qual certas cavidades são preenchidas continuamente com graute, contendo armaduras envolvidas o suficiente para absorver os esforços calculados, além daquelas armaduras com finalidade construtiva ou de amarração".

Na década de 1950, segundo Barbosa (2000, p.1), a utilização da alvenaria estrutural voltou a aumentar. Começaram, então, as construções maiores, tais como edifícios altos, uma vez que, se aumentou o interesse na busca de um sistema racionalizado, e assim, se intensificaram as pesquisas nessa área.

Com a disseminação da informática ocorrida no final da década de 1980 e principalmente em função do grande aumento da capacidade de armazenamento, gerenciamento e processamento de dados apresentados pelos computadores de pequeno porte, disponibilizou-se 
ao Engenheiro de Estruturas o acesso a equipamentos e programas computacionais que possibilitam uma análise estrutural baseada em modelos mais refinados, proporcionando um aumento da confiabilidade e diminuição de custos dos projetos e das construções (WAIDEMAN, 2004, p.1).

Nesse cenário, a análise estrutural estática tem como objetivo principal a determinação da distribuição e da intensidade das tensões e dos deslocamentos que se manifestam em qualquer sistema estrutural, quando o mesmo é submetido a um carregamento qualquer, cuja intensidade, direção e sentido não variem com o tempo (FAGLIONI, 2006, p.13). Desse modo, o campo de tensões calculado deve apresentar um equilíbrio entre as forças internas e externas, assim como deslocamentos contínuos (ALBERTINI, 2009, p. 18).

Para possibilitar essas análises de forma mais rápida, foram desenvolvidos modelos numéricos, de modo que, dentre os existentes e estudados na atualidade destaca-se o Método dos Elementos Finitos. Esse método, assim como os outros, é utilizado juntamente com programas computacionais e, dessa forma, se transforma em uma ferramenta que agiliza o processo de cálculo, comparado com métodos analíticos, e apresenta resultados, com precisão satisfatória, nos problemas relacionados à Engenharia Estrutural (ALTRAN, 2010, p.15).

Conforme Mata (2009, p. 45), há uma grande evolução tecnológica mundial, associada ao conhecimento das propriedades mecânicas da alvenaria, que proporciona condições para que as construções sejam seguras e ao mesmo tempo econômicas, mas, apesar disso, este potencial não tem sido utilizado em toda a sua extensão, principalmente, em prédios de alvenaria estrutural, devido ao incompleto conhecimento das propriedades das unidades e argamassas, incluindo o comportamento da ligação entre esses dois materiais.

Neste estudo foi analisado o comportamento das paredes (painéis) de alvenaria com o objetivo principal de determinar a resistência da mesma sob uma combinação específica de esforços. Espera-se, com isso, que os resultados das simulações numéricas propostas possam contribuir para a otimização do sistema da alvenaria estrutural, tornando o mesmo mais eficiente, isto é, melhorar a qualidade da execução e reduzir custos.

Segundo Altran (2010, p.14), ao longo dos últimos anos, as obras de alvenaria estrutural têm uma grande aceitação no mercado, por apresentar diversas vantagens, entre elas destacamse: redução dos custos finais da obra, rapidez na execução, diminuição da mão-de-obra e simplificação das técnicas de execução. Por este motivo, esse sistema tem sido apoiado por construtoras buscando aumentar o nível de industrialização de suas obras. 


\section{METODOLOGIA}

Foi desenvolvido através do SAP $2000^{\circ}$ (versão free, limitada), um programa estrutural existente no mercado, um modelo de painel de alvenaria estrutural, submetidos a esforços externos. Este painel já existe em outra bibliografia onde foi ensaiado experimentalmente e analisado numericamente através do programa estrutural DIANA ${ }^{\circledR}$, possibilitando a comparação dos resultados.

O SAP2000 tem como base o Método dos Elementos Finitos (MEF). O MEF consiste na subdivisão de um sistema estrutural em pequenas regiões, chamadas elementos finitos, estes elementos são interconectados por meio de pontos nodais. Os parâmetros básicos desconhecidos são os deslocamentos desses nós. Em projetos de engenharia civil os métodos numéricos ocupam lugar de destaque uma vez que tornam a análise estrutural mais rápida e menos dispendiosa.

Na sequência, foi elaborado o modelo do painel no programa. Este painel foi escolhido na bibliografia existente, utilizando dimensões e aplicação de carregamentos do modelo já ensaiado.

Os resultados encontrados puderam ser outorgados com uma comparação aos resultados encontrados por Mata (2011) na bibliografia de referência.

\section{RESULTADOS E DISCUSÕES}

Neste trabalho foi feita a modelagem numérica de um painel de alvenaria de $159 \mathrm{~cm} x$ $119 \mathrm{~cm}$, utilizando-se o método dos elementos finitos, dividindo o mesmo em 28 elementos, no programa SAP $2000^{\circ}$, e aplicando-se uma carga de pré-compressão uniformemente distribuída em sua superfície de 2,5MPa, representado a carga resultante de uma viga metálica e laje de concreto, além da aplicação de uma força horizontal na parte superior na viga metálica 50KN, conforme ilustra a Figura 1. Os valores considerados foram os máximos aproximados encontrados nos resultados experimentais e numéricos do trabalho de referência utilizado de Mata (2011).

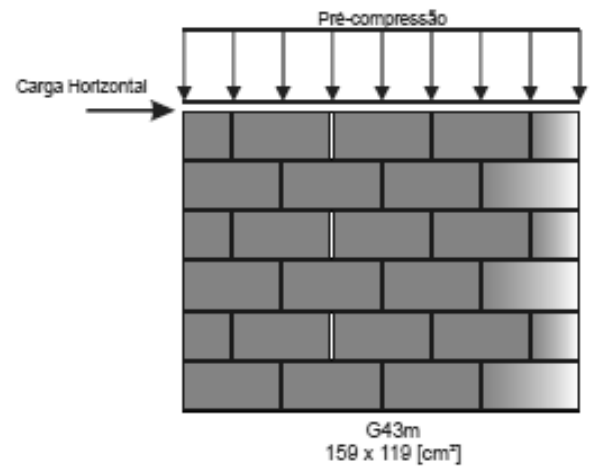

Figura 1. Geometria do painel e pontos de aplicação de carga.

(Fonte: MATA \& RAMALHO, 2009, p. 48). 
As propriedades mecânicas utilizadas para os blocos de concreto e juntas de argamassa estão relacionadas nas Tabelas 1 e 2, a seguir, conforme Mata (2011, p. 137 e 139).

Tabela 1. Propriedades mecânicas dos blocos.

\begin{tabular}{c|c|c|c|c|c|c|c}
\hline $\begin{array}{c}f_{c} \\
(\mathrm{MPa})\end{array}$ & $\begin{array}{c}E \\
(\mathrm{MPa})\end{array}$ & $v$ & $\begin{array}{c}G_{c} \\
(\mathrm{MPa} \cdot \mathrm{mm})\end{array}$ & $\begin{array}{c}f_{b t} \\
(\mathrm{MPa})\end{array}$ & $\begin{array}{c}G_{f}^{I} \\
(\mathrm{MPa} \cdot \mathrm{mm})\end{array}$ & $\beta$ & $\begin{array}{c}\text { Largura da banda } \\
\text { de fissura }\end{array}$ \\
\hline 12,86 & 7586 & 0,37 & 19,94 & 0,99 & 0,05 & 0,03 & $1 \mathrm{~mm}$ \\
\hline
\end{tabular}

Onde: $f_{c}$ é a resistência média à compressão axial, $v$ é o coeficiente e Poisson, $G_{c}$ é a energia de fraturamento à compressão, $\mathrm{f}_{b t}$ é resistência média à tração calculada em relação a área bruta do bloco, $G_{f}^{I}$ é a energia de fraturamento à tração, $\beta$ é o fator de retenção do cisalhamento.

Tabela 2. Propriedades mecânicas das juntas de argamassa.

\begin{tabular}{c|c|c|c|c|c|c|c|c|c|c|c|c}
\hline & $\begin{array}{c}f_{t} \\
(\mathrm{MPa})\end{array}$ & $\begin{array}{c}k_{n} \\
\left(\mathrm{~N} / \mathrm{mm}^{3}\right)\end{array}$ & $\begin{array}{c}k_{s} \\
\left(\mathrm{~N} / \mathrm{mm}^{3}\right)\end{array}$ & $\begin{array}{c}G_{f}^{I} \\
(\mathrm{MPa} \cdot \mathrm{mm})\end{array}$ & $\begin{array}{c}f_{c} \\
(\mathrm{MPa})\end{array}$ & $\begin{array}{c}\mathrm{G}_{c} \\
(\mathrm{MPa} \cdot \mathrm{mm})\end{array}$ & $\begin{array}{c}\varepsilon_{c} \\
\left(10^{-3}\right)\end{array}$ & $C_{s s}$ & $\begin{array}{c}f_{\mathrm{vo}} \\
(\mathrm{MPa})\end{array}$ & $\tan (\varphi)$ & $\tan (\psi)$ & $\begin{array}{c}G_{f}^{I} \\
(\mathrm{MPa} \cdot \mathrm{mm})\end{array}$ \\
\hline $\mathrm{A} 1$ & 0,085 & 34,38 & 161,82 & 0,005 & 8,68 & 14,41 & 3,7 & 2 & 0,235 & 0,624 & 0 & 0,02 \\
\hline $\mathrm{A} 2$ & 0,093 & 58,66 & 118,71 & 0,005 & 7,92 & 11,64 & 5,4 & 2 & 0,208 & 0,612 & 0 & 0,09 \\
\hline
\end{tabular}

Onde:

$f_{t}$ é a resistência à tração da junta;

$\mathrm{k}_{\mathrm{n}}$ e $\mathrm{k}_{\mathrm{s}}$ são módulos de rigidez elástica normal e transversal, respectivamente;

$G_{f}^{I}$ é a energia de fratura do modo I;

$\mathrm{f}_{\mathrm{c}}$ é a resistência à compressão média de prisma de 3 blocos;

$\mathrm{G}_{\mathrm{c}}$ é a energia de fratura à compressão de prisma de 3 blocos;

$\varepsilon_{\mathrm{c}}$ é a deformação correspondente ao pico da tensão de compressão do prisma;

$\mathrm{C}_{\mathrm{ss}}$ é o controle de contribuição das tensões de cisalhamento na ruptura;

$f_{\text {vo }}$ é a tensão de cisalhamento na ausência de compressão;

$\tan (\phi)$ é o coeficiente de atrito;

$G_{f}^{I I}$ é a energia de fratura do modo II. 
Os resultados foram comparados com os modelos da bibliografia que segue.

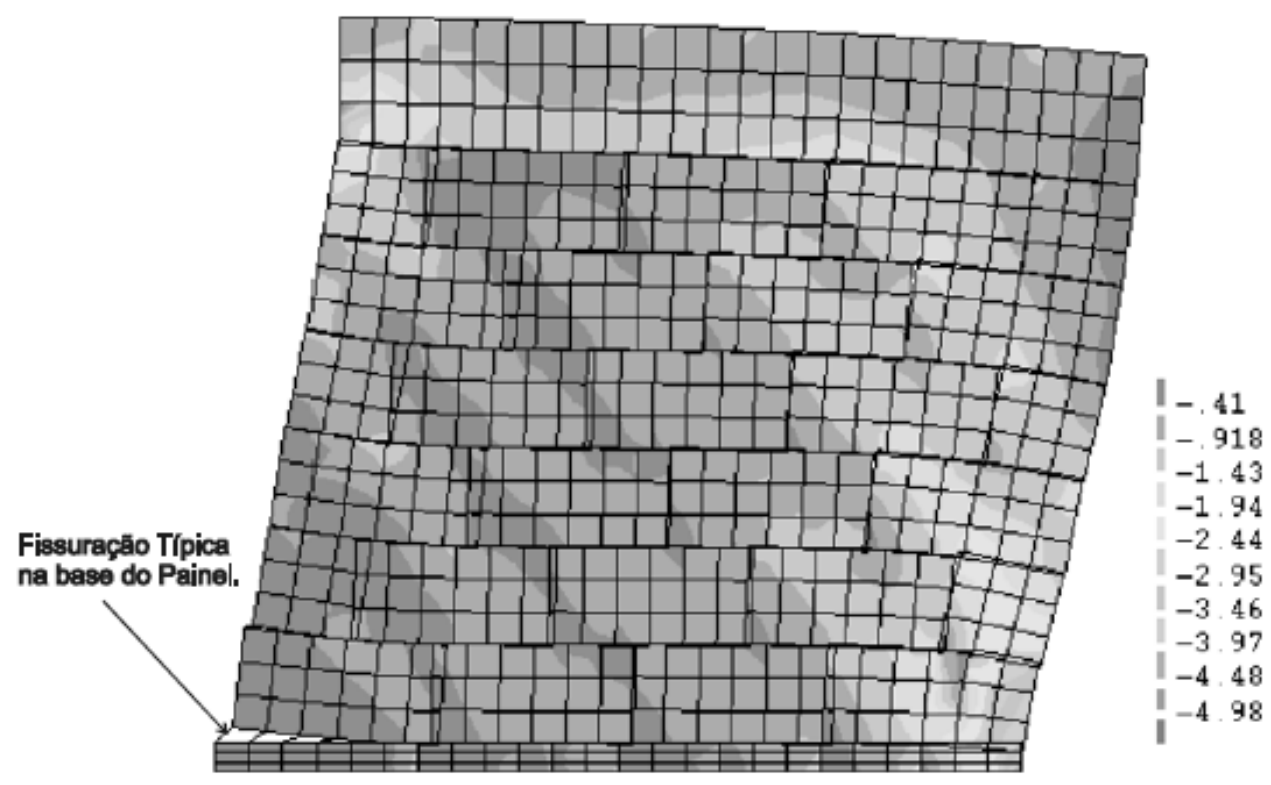

Figura 2. Deformação e tensões principais (em MPa) - painel PCA1.

(Fonte: MATA, 2011, p. 143).

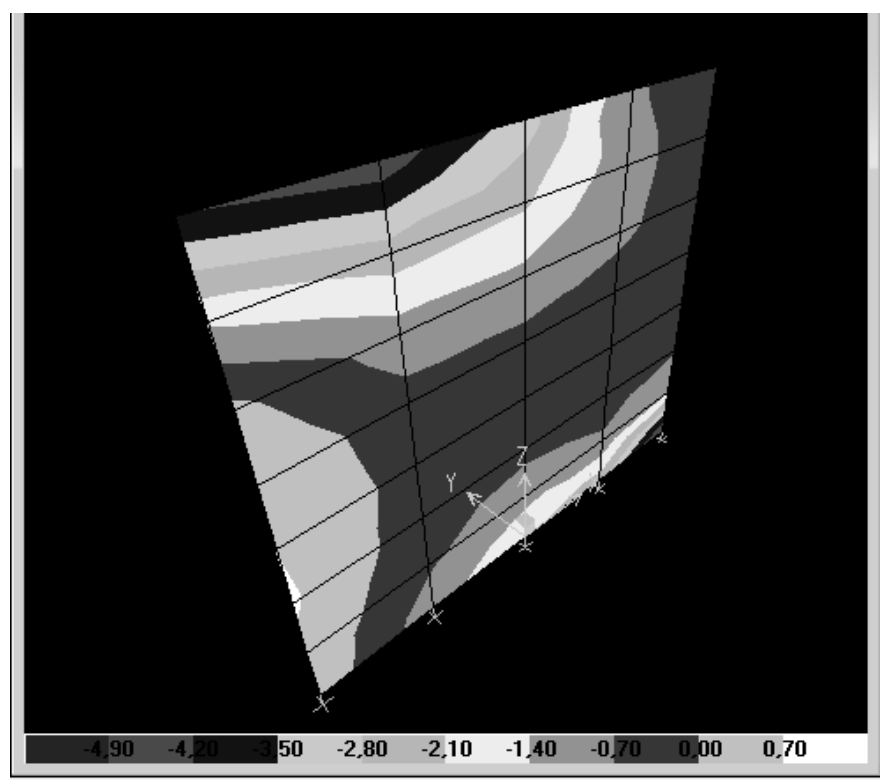

Figura 3. Tensões principais (em MPa) painel. 


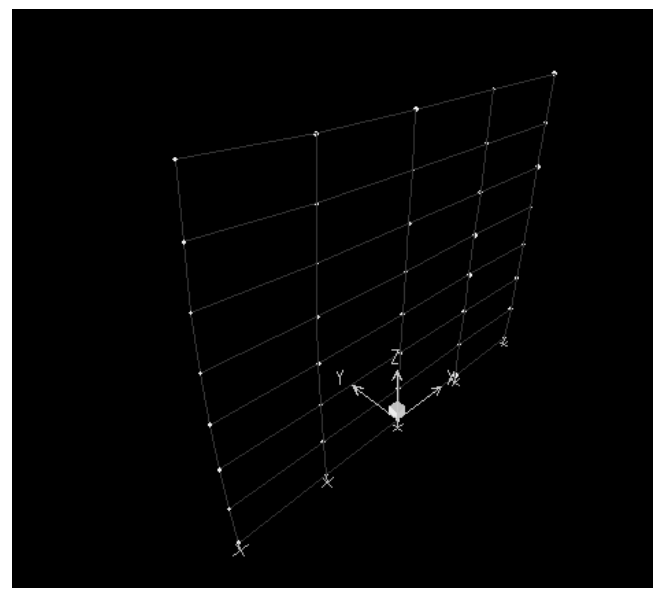

Figura 4. Deformações do painel.

Como é possível observar, os resultados das tensões e deformações encontradas (Figura 3 e 4), assemelham-se aos obtidos por Mata (2011) - ver Figura 2.

Na região superior onde foi aplicada a força horizontal e na região oposta próxima ao apoio (inferior) são os locais onde ocorrem as maiores tensões de compressão, entre $-3,5$ a $-4,9 \mathrm{Mpa}$, verificando a ocorrência dos maiores deslocamentos nesse ponto. No apoio do canto esquerdo, ocorrem tensões de tração com tendência a sofrer fissuras de tração por flexão na base, que são de aproximadamente 0,70 MPa.

\section{CONCLUSÃO}

Neste trabalho foi desenvolvido um estudo do comportamento de painel de alvenaria estrutural, visando uma análise por meio de modelagem numérica simplificada (devido às restrições do uso da versão limitada do programa SAP2000') e comparando os resultados encontrados por Mata (2011), que desenvolveu um estudo por meio de comparações experimentais e numéricas. Apesar dessa limitação, os resultados encontrados foram satisfatórios aproximando-se do comportamento esperado do trabalho utilizado como referência.

Os estudos referentes à alvenaria estrutural vêm se intensificando nos últimos anos, desse modo, as pesquisas existentes estão ainda em fase de desenvolvimento. Neste cenário, o estudo desenvolvido, contribui para o início das pesquisas sobre este assunto na UNOESTE. Futuramente, outros trabalhos poderão aprimorar este estudo, melhorando os resultados encontrados, por exemplo, refinando mais a malha dos elementos divididos, acrescentando análises não-lineares, entre outros. 


\section{REFERÊNCIAS}

ALBERTINI, M. M. Análise do comportamento experimental e numérico de prismas de alvenaria estrutural utilizando o elemento finito prismático regular parabólico. 2009. 158f. Dissertação (Mestrado em Engenharia Civil) - Faculdade de Engenharia, Universidade Estadual Paulista, Ilha Solteira, 2009. 158p.

ALTRAN, D. A. Procedimento numérico para análise de vigas de alvenaria estrutural submetidas à flexão simples. 2010. Dissertação (Mestrado em Engenharia Civil) - Faculdade de Engenharia, Universidade Estadual Paulista, Ilha Solteira, 2010. 88p.

ASSOCIAÇÃO BRASILEIRA DE NORMAS TÉCNICAS. NBR-10837: cálculo de alvenaria estrutural de blocos vazados de concreto. Rio de Janeiro: Associação Brasileira de Normas Técnicas, 1989. 20p.

BARBOSA, P. C. Estudo da interação de paredes de alvenaria estrutural com vigas de concreto armado. 2000. 110f. Dissertação (Mestrado)- Escola de Engenharia de São Carlos, Universidade de São Paulo, São Carlos, 2000, 110p.

FAGLIONI, A. F. Análise não-linear física de vigas de concreto armado utilizando o elemento finito prismático regular linear associado ao de barra. 2006. 122f. Dissertação (Mestrado em Engenharia Civil)- Faculdade de Engenharia, Universidade Estadual Paulista, Ilha Solteira, 2006, 122p.

MATA, R. C.; RAMALHO, M. A. Análise experimental e numérica do comportamento de junta da alvenaria. Cadernos de Engenharia de Estruturas, São Carlos, v. 11, n. 53, p. 45-49, 2009.

MATA, R.C. Análise experimental e numérica do comportamento de junta em painéis de contraventamento de alvenaria estrutural. 174p. Tese (Doutorado) - Escola de Engenharia de São Carlos, Universidade de São Paulo, São Carlos, 2011.

WAIDEMAN, L. Análise dinâmica de placas delgadas utilizando elementos finitos triangulares e retangulares. 2004. 153f. Dissertação (Mestrado em Engenharia Civil) - Faculdade de Engenharia, Universidade Estadual Paulista, Ilha Solteira, 2004. 